Ciencia y Educación, Vol. 6, No. 1, enero-abril, 2022

ISSN (impreso): 2613-8794・ISSN (en línea): 2613-8808

DOI: https://doi.org/10.22206/cyed.2022.v6i1.pp43-61

\title{
Formar directivos para la escuela real. Un enfoque basado en el análisis de la práctica en grupos
}

\author{
Training principals for the real school. \\ A practice-based analysis in groups
}

\author{
Marita Sánchez-Moreno ${ }^{a}$ ORCID: 0000-0002-5744-3959 \\ Julián López-Yáñez ORCID: 0000-0001-8978-964X
}

Recibido: 29/07/2021 • Aprobado: 16/11/2021

\begin{abstract}
Cómo citar: Sánchez-Moreno, M., \& López-Yánez, J. Formar directivos para la escuela real. Un enfoque basado en el análisis de la práctica en grupos. Ciencia y Educación, 6(1), 43-61. https://doi.org/10.22206/cyed.2022.v6i1.pp43-61
\end{abstract}

\section{Resumen}

El artículo presenta un programa de formación continua de directores/as escolares, junto a los principales resultados del proyecto de investigación que se desarrolló en paralelo para determinar su alcance y eficacia. El programa adoptó un enfoque de coaching grupal, implicando la retroalimentación mediante instrumentos específicos y a partir de casos elaborados por los participantes sobre situaciones problemáticas experimentadas en su práctica directiva. En el proyecto de investigación se evaluó la satisfacción de los participantes y el rendimiento de los grupos según su ajuste a la estructura del programa. Además, se realizó un análisis primario de la interacción dentro de los grupos, así como sendos análisis secundarios sobre (a) los sesgos y las dificultades comunicativas encontrados por los grupos y las maneras de afrontarlos, y (b) la construcción de conocimiento práctico por parte de los participantes. Se reúnen por primera vez los desarrollos principales del programa con el objeto de establecer conclusiones que nos permitan avanzar en la búsqueda de estrategias eficaces para la formación de directivos escolares.

Palabras clave: administrador de la educación, apoyo pedagógico, dinámica de grupos, capacitación, liderazgo, conocimiento.

\footnotetext{
a Universidad de Sevilla, España. Correo-e: marita@us.es

b Universidad de Sevilla, España. Correo-e: lopezya@us.es
}

\begin{abstract}
The paper presents a program for school principals' in-service preparation and the main results of the research project implemented in order to analyse its scope and effectiveness. The program adopted a group coaching focus, which involved the feedback to the participants by means of both, specific tools, and cases about problematic experiences faced by them. The research project assessed participants' satisfaction with the program, and group performance according to their fitting to the programme goals and structure. Additionally, a primary qualitative analysis was carried out about group interaction, which led to the research team to a new, double, secondary analysis about: (a) the communicational biases and difficulties faced by the groups -and the strategies implemented to overcome such difficulties-; and (b) participants' building process of professional knowledge. The fact that the paper collects for the first time the main pieces of the research project allows us to discuss relevant conclusions in search of useful strategies for school principals' preparation.
\end{abstract}

Keywords: Principals, coaching, group dynamics, training, leadership, knowledge. 


\section{Introducción}

Este artículo hace un balance del desarrollo y de los resultados de un programa de formación de directivos escolares basado en el análisis de la práctica, la retroalimentación y el coaching grupal. Las diferentes fases del programa -diseño e implementación de una primera versión, pilotaje, mejora e implementación de la segunda versión, evaluación y análisis primario y secundario- se llevaron a cabo bajo dos proyectos financiados que, en conjunto, abarcaron cinco años completos: 2013 a 2017. La primera versión del programa se desarrolló entre enero de 2013 y diciembre de 2014, bajo un proyecto internacional financiado por la Unión Europea en el que participaron 9 países junto con el nuestro, España ${ }^{1}$ (López-Yáñez et al., 2018). En la fase siguiente y mediante otro proyecto de investigación nacional se llevó a la práctica una segunda versión del programa, en el ámbito de la Comunidad Autónoma de Andalucía, en colaboración con las autoridades educativas regionales ${ }^{2}$.

La investigación que se desarrolló en paralelo con el programa de formación permitió: (a) evaluar la satisfacción de los participantes con el programa; (b) determinar el grado de ajuste de los 14 grupos participantes a los objetivos y la estructura del programa; (c) analizar la comunicación en el interior de los grupos y, en particular, los sesgos y las dificultades que afrontó el proceso formativo; y (d) la construcción de conocimiento profesional por parte de los participantes. Todos estos aspectos han sido analizados con más detalle del que este texto permite en diferentes artículos, algunos ya publicados y otros en elaboración. Sin embargo, el presente artículo permitirá analizar y discutir por primera vez una panorámica general y completa del proceso.

\section{Marco teórico}

Nuevos tiempos, nuevo enfoque de la formación de directivos escolares

La dirección escolar ha sido señalada por la investigación educativa como uno de los factores clave de la mejora escolar que la sociedad actual insistentemente reclama (Day \& Sammons, 2013; Pont et al., 2009). Por otro lado, el aumento del conocimiento disponible sobre los factores a los que se puede asociar el éxito escolar ha traído nuevas funciones y demandas muy específicas para los directivos. Una buena gestión, aunque necesaria, ya no resulta suficiente. Muchas voces reclaman más papel para el liderazgo entre las funciones de la dirección (Day et al., 2008), especialmente en sus variantes pedagógica (Grissom et al., 2021) y distribuida (Harris et al., 2007; Spillane $\&$ Healey, 2010). Pero también se les reclama que sean capaces de construir capacidad de cambio en sus escuelas (Harris et al., 2007), o que las transformen en verdaderas comunidades de aprendizaje (Stoll \& Kools, 2017).

Sin duda, esto ha supuesto un notable incremento en la complejidad asociada a la función directiva (Iranzo-García et al., 2018; Retna, 2015). No solo se requiere ahora más formación, sino que esta ha de venir con un nuevo enfoque, ya que la complejidad de las demandas apela a una formación más sofisticada y variada (Schleicher, 2012; Forde et al., 2013; Walker et al., 2013).

Muchos países de la OCDE han hecho avanzar en los últimos años sus políticas orientadas al desarrollo profesional de los directores escolares (Pont et al., 2009). La investigación también ha contribuido a ampliar las modalidades y estrategias empleadas en estos programas, tanto para aspirantes a director, nóveles o directores con experiencia (Huff et al., 2013). Sin embargo, los sistemas educativos latinoamericanos se han mostrado, en general, poco ágiles a la hora de responder al reto de la formación de sus directivos. Aún resulta poco frecuente encontrar modalidades de formación diferentes de la tradicional, eminentemente teórica, impartida por expertos y basada habitualmente en una colección de prescripciones 'saludables' que, sin embargo, difícilmente encuentran el camino hacia un impacto real sobre la práctica de la dirección (Weinstein et al., 2018).

No obstante, en el plano internacional, una nueva generación de programas de formación se viene ensayando con tres focos principales: 
a) Un foco sobre el análisis de la práctica, con frecuencia a partir de casos sobre incidentes críticos o situaciones problemáticas experimentadas por los participantes (Hallinger $\&$ Bridges, 2017; Hallinger \& Snidvongs, 2005; Zhang \& Brundrett, 2010).

b) Un foco sobre el conocimiento y la emocionalidad de los/as directores/as, que da lugar a estrategias que se proponen mejorar la práctica fortaleciendo su autoconciencia como líderes, así como sus creencias y su conocimiento implícito sobre la gestión y el liderazgo (Huber, 2013), lo que a menudo tiene un efecto positivo sobre su autoeficacia (Reichard et al., 2017).

c) Un foco sobre la comunicación que enfatiza la cooperación y el diálogo constructivo entre directores para compartir y analizar no solo sus problemas, sino también sus mejores prácticas (Piggot et al., 2011). Los expertos externos no son la única fuente de conocimiento. Los directivos también pueden aprender de sí mismos y de sus colegas más experimentados o que han desarrollado una carrera notable y exitosa en la profesión (Forde et al., 2013).

Las nuevas estrategias formativas encuentran sustento teórico en los principios de la andragogía, la disciplina que estudia el aprendizaje en los adultos. Sabemos que los adultos necesitan razones convincentes para desear aprender algo; que no les vale únicamente con motivaciones extrínsecas, sino que las necesitan también intrínsecas; que necesitan un importante margen de control o autorregulación; que su aprendizaje se construye mejor sobre sus experiencias previas; que necesitan aplicar inmediatamente lo aprendido; y que el proceso es más exitoso cuando se centra en los problemas que cuando lo hace en el contenido (Giannoukos et al., 2015; Knowles, 1984; Knowles et al., 2015; Merriam \& Bierema, 2014; Rothes et al., 2017; Youde, 2018).

Por tanto, la experiencia es el recurso fundamental en el aprendizaje adulto. Esta sirve -de manera aún más poderosa que en niños y jóvenes- para dar sentido a los nuevos eventos (Bagnall, 2017; Illeris, 2017).
Concretamente, este proceso de aprendizaje experiencial se realiza en seis etapas: motivación, orientación, implicación, actividad, reflexión y adaptación (Dyke, 2017; Merriam \& Bierema, 2014).

Lo cierto es que, de manera creciente, los programas de formación de directivos escolares asumen que no se trata tanto de 'enseñar' a los directores, como de que estos 'aprendan' (Whitmore, 2004), para lo cual adoptan estrategias tan variadas como la autoevaluación, la supervisión clínica, la mentoría, la retroalimentación o el coaching (Orr, 2011; Schleicher, 2012; Walker et al., 2013). A continuación, se abordan las dos últimas, ya que constituyen el eje del programa de formación desarrollado.

La retroalimentación y el coaching como estrategias para el desarrollo del liderazgo educativo

El coaching es una estrategia que asume que el aprendizaje de habilidades y conocimientos técnicos ha de ser compatible con el crecimiento personal y el bienestar dentro de la profesión (Hargrove, 2008; Whitmore, 2004). Como afirman Bloom et al. (2005), refiriéndose al blended coaching (mixto), se aprende a hacer (algo) al mismo tiempo que a ser (alguien). Es decir, ambas facetas se construyen dialógicamente, a partir del autoanálisis de los participantes conducido por expertos (Aas \& Vavik, 2015; Huff et al., 2013). Desde un punto de vista amplio, el coaching se asocia al apoyo deliberado y especializado a los sujetos para ayudarlos a clarificar o alcanzar sus metas (Bloom et al., 2005), afrontar los desafíos que les plantean las organizaciones en las que trabajan (Lofthouse, 2019) y, al mismo tiempo, aumentar la percepción de reconocimiento y el compromiso con su trabajo (Creasy \& Paterson, 2015).

Pero, de la práctica se aprende mejor en equipo. Diversos estudios han señalado el grupo como medio idóneo de la reflexión y el aprendizaje profesional (Lofthouse, 2019; Van Knippenberg \& Schippers, 2007; Youde, 2018). Por eso vemos crecer actualmente el coaching grupal, que aprovecha el potencial que tiene la comunicación entre profesionales que afrontan similares problemáticas para generar y 
transferir nuevo conocimiento, así como profundizar en lo que ya se conoce (Britton, 2015; Robertson, 2008; Thornton, 2010). Según Dyke (2014), se trata de provocar en los participantes una autocomprensión más profunda -involucrando sentimientos y emociones- y un análisis también más profundo y comprometido de su práctica.

El coaching grupal encuentra sustento teórico en las teorías del aprendizaje situado, que reclaman atención al contexto social del aprendiz como condicionante fundamental de su proceso de aprendizaje (Cosner et al., 2018; Lave \& Wenger, 1991), así como en las teorías del capital social y las redes sociales, que justifican la importancia de la interacción social como mecanismo de aprendizaje (Cullen-Lester et al., 2017; Daly et al., 2010; Wright \& Adam, 2015). Estas bases teóricas son compartidas con la perspectiva de las comunidades profesionales de práctica (Stoll \& Kools, 2017; Wenger, 1998), con la cual también ha establecido una fértil influencia.

El coaching grupal requiere mecanismos para el desarrollo en comunidad de la reflexividad (Camburn \& Han, 2017; Ellis et al., 2014; Konradt et al., 2016; Merriam \& Bierema, 2014). Uno de estos mecanismos es la retroalimentación (Lochmiller, 2014; Orr \& Orphanos, 2011). La retroalimentación se encarga de colocar la práctica experimentada por los participantes en el centro del proceso de formación y les ayuda a reflexionar sobre su perfil y sus habilidades para el liderazgo (Goff et al., 2014; Petridou et al., 2017; Serrat \& Badía, 2015).

Por lo general, la retroalimentación proviene de herramientas específicas - principalmente cuestionarios dirigidos a los participantes y otros miembros de sus comunidades educativas- que obtienen y devuelven información sobre la práctica, las características o las habilidades de los directivos (Walker \& Smither, 2006). Por ejemplo, el programa de formación que se presenta y analiza en este artículo utilizó un cuestionario propio sobre el perfil de liderazgo de los participantes (Huber \& Hiltmann, 2011), así como un cuestionario sobre algunas características organizativas de sus centros, el cual fue respondido por ellos mismos y el resto del profesorado.
Otras veces la retroalimentación se realiza a partir de breves casos elaborados y narrados por los miembros del grupo a partir de sus vivencias ante problemas profesionales experimentados en su práctica (Sawyer et al., 2000), casos que posteriormente son analizados por el grupo. Varios programas de formación de líderes escolares han reportado una recepción muy favorable por parte de los participantes de la recreación de situaciones problemáticas, acompañada de posterior retroalimentación sobre su actuación (Ming Yu, 2018; Salazar et al., 2013; Schleicher, 2012).

La retroalimentación facilita la reflexión situada, contextualizada o reflexión en la acción: una mirada a las experiencias pasadas, resignificándolas y analizándolas retrospectivamente, para aprender tanto de lo que funcionó como de aquello en lo que se fracasó (Schön, 1991). Precisamente, para Wright \& Adam (2015, p. 441), la práctica reflexiva implica criticar, repensar y resignificar acciones previamente realizadas, a menudo con la ayuda de amigos críticos, lo cual requiere un clima de apertura y confianza mutua (Bloom et al., 2005). Para estos investigadores, el grupo de iguales puede funcionar como tal amigo crítico, ayudando a los protagonistas a identificar efectos de sus acciones no considerados previamente.

\section{El grupo como generador de conocimiento sobre la dirección escolar}

La reflexividad, espoleada por la retroalimentación de otros compañeros y la dirección especializada del facilitador, convierte al grupo de formación en un generador de conocimiento, antes que en un mero receptor. Como se vio en la sección anterior, la reflexión sobre la práctica resulta un medio idóneo para la mejora sostenible del liderazgo educativo (Silva et al., 2017). No es casualidad que las ciencias de la educación estén interesándose de manera creciente por los procesos de aprendizaje grupal o colaborativo, que ponen el énfasis en la construcción del conocimiento mediante la comunicación estructurada y la reflexión (Fernández-Sánchez \& Valverde-Berrocoso, 2014; Hernández-Sellés et al., 2014). 
Pero reflexionar constituye una actividad compleja y comprometida cuando se realiza en grupo, ya que supone el esfuerzo no solo de entender una situación, sino también de entenderse uno mismo en tanto actor en esa situación (Ming Yu, 2018). Un dispositivo de reflexión es útil únicamente cuando hace circular la comunicación de tal manera que los participantes pueden contemplar situaciones experimentadas personalmente desde nuevas perspectivas (Kramer, 2018) y, como consecuencia, pueden plantearse nuevas respuestas. Para lograrlo, los grupos de formación han de contar con una cuidadosa planificación de tareas estructuradas (Lofthouse, 2019), usar recursos como incidentes críticos sobre los que reflexionar (Ming Yu, 2018) y dar una preparación especializada al facilitador, cuyo papel es decisivo (Youde, 2018).

Sin embargo, poco se sabe aún acerca de las dificultades y disfunciones comunicativas que este proceso puede atravesar y, sobre todo, de las estrategias que los grupos exitosos utilizan para superarlas, aunque sí se cuenta con una incipiente literatura científica sobre las condiciones que requiere la reflexividad en grupos pequeños $y$, en particular, en los grupos de formación (James-Ward, 2011; Konradt et al., 2016). Por eso este seleccionó y adaptó el esquema de Schippers et al. (2014) para el análisis de las condiciones y dificultades de la reflexión en grupo. El sistema completo de categorías de análisis se detalla más adelante en una subsección específica de la metodología y, en concreto, en la Figura 2.

Por otro lado, también cabe y resulta interesante investigar el producto de la actividad reflexiva grupal, esto es, el conocimiento elaborado por los participantes durante la interacción grupal. El dominio de cualquier actividad profesional exige la adquisición de una notable cantidad de conocimiento, no solo explícito y formal, sino también y sobre todo tácito, experiencial, adquirido informalmente a través de la práctica (Nestor-Baker \& Hoy, 2001; Pont et al., 2009; Zhang \& Brundrett, 2010). Es precisamente este conocimiento tácito el que permite a un experto identificar recurrencias o patrones y seleccionar la norma, el principio o la regla-conocimiento codificado- que se debe aplicar en cada momento (Devos \& Bouckenooghe, 2009). El conocimiento tácito adopta múltiples formas, como creencias, metáforas, teorías-en-acción o implícitas, repertorios o modelos mentales, representaciones, imágenes o valores (Begley, 2006; Botha, 2014; Lazaridou, 2007; Pozo et al., 2010). Los dispositivos de formación basados en la reflexión grupal pueden contribuir decisivamente a transformar-codificar- el conocimiento tácito en explícito.

Nuestro estudio analizó el conocimiento práctico que dos grupos participantes en el programa formativo pusieron en juego cuando se enfrentaron a situaciones desafiantes en forma de casos narrados por sus compañeros. Para eso utilizamos sucesivamente los marcos conceptuales de Poggi (2001) y Lazaridou (2008), los cuales serán explicados en la sección de metodología.

\section{Metodología}

El problema que dio lugar a esta investigación, realizada mediante dos proyectos financiados sucesivamente, fue la ausencia en nuestro ámbito de una sólida tradición de programas de formación de directivos escolares basados en el análisis de la práctica y el aprendizaje en grupo de iguales. Para abordar este problema se diseñó, pilotó, mejoró y aplicó una versión definitiva de un programa específico y original de formación. Al mismo tiempo, de forma paralela al programa de formación, se llevó a cabo un proceso de investigación con los siguientes objetivos: (a) evaluar el programa; y (b) profundizar en nuestro conocimiento sobre los procesos implicados en el coaching grupal como estrategia formativa. Esta sección describirá los procedimientos seguidos para alcanzar ambos objetivos.

\section{Participantes}

En el programa de formación participaron noventa y cuatro directores y directoras con diferente nivel de experiencia profesional y adscritos a centros educativos de diferentes tipologías. Se organizaron grupos que estaban compuestos inicialmente por 7 directores/as, uno de 
los cuales debía ejercer el papel de coach o coordinador/ facilitador del grupo, para lo que previamente recibió formación específica. Finalmente, fueron catorce los grupos de formación participantes, ubicados en las ocho provincias andaluzas. La Junta de Andalucía colaboró en el desarrollo del programa y las sesiones de formación se llevaron a cabo en Centros de Profesorado dependientes de la Consejería de Educación.

Los participantes tenían edades entre los 34 y los 67 años y su experiencia docente oscilaba entre 8 y 39 años, aunque el $75 \%$ de ellos tenía una experiencia docente de más de 18 años. La distribución por sexo resultó igualitaria: 50,7\% hombres y $49,7 \%$ mujeres. El 38\% tenía experiencia en cargos directivos anterior al puesto que en ese momento ejercían. La distribución en función del tipo de centro de procedencia era la siguiente: 46,6\% Centros de Educación Infantil y Primaria o solo de Educación Infantil; 39,7\% Institutos de Educación Secundaria; 5,5\% Centros del Profesorado; 4,1\% Centros de Educación Especial, y el resto centros de Educación de Personas Adultas, Escuelas de Idiomas o Escuelas de Arte. Un 40,9\% de los centros se ubicaba en zonas de nivel socioeconómico bajo, el $57,6 \%$ en zonas de nivel medio y $1,5 \%$ en zonas de nivel alto. Como dato interesante, el 70\% de los directores de centros en zonas de bajo nivel sociocultural correspondió a mujeres.

\section{El programa "Grupos de autoformación para la direc- ción escolar"}

El programa de formación incluyó 7 sesiones presenciales de 3 horas de duración, organizadas en cuatro partes, y una serie de actividades no presenciales (Figura 1). En la primera parte (primera sesión) el grupo se constituye con un foco especial en la construcción de una relación de confianza entre los participantes (Bloom et al., 2005), que deben sentirse seguros para expresar y recibir críticas y comentarios de sus compañeros (Whitmore, 2004). Con tal objeto, los coordinadores presentan el programa y cada participante describe el centro escolar donde trabaja y relata su trayectoria como directores al resto de los participantes. Mientras tanto, los coordinadores hacen preguntas que ayudan a construir vínculos entre los participantes.

La sesión 2 -«Taller de Competencias»- y la 3 - «Taller Contexto»-constituyen la segunda parte del programa. Previamente, los participantes han debido responder a dos instrumentos de autoevaluación online - «Perfil de Competencias para la Dirección Escolar» (CPSM) y «Perfil del Centro Escolar y su Contexto» (PCEC) - y habrán recibido un informe de retroalimentación que les ayuda a identificar y reflexionar sobre su perfil de liderazgo y el impacto que este tiene sobre el resto de la comunidad educativa (Robinson et al., 2008). Una vez en las sesiones presenciales, el coordinador y el resto del grupo analizan conjuntamente los resultados de los informes de cada participante y le ayudan a interpretarlos, poniendo el foco especialmente sobre la relación entre ambos perfiles -competencias individuales y contexto organizativomediante la formulación de preguntas.

Las tres sesiones de la tercera parte del programa (4, 5 y 6) siguen la misma estructura y se denominan «bajo los focos». Dos participantes en cada sesión describen consecutivamente a sus compañeros y analizan con ellos una situación problemática experimentada en su práctica profesional. El programa prevé una secuencia de indagación y análisis altamente estructurada para dirigir la discusión del grupo sobre la actuación del director bajo los focos ante esa situación. La secuencia se inicia con tres tandas consecutivas de preguntas: «puntuales», «horizontales» (para completar la información) y «verticales» (para promover el análisis). Tras la recogida de información regulada por el coordinador, el grupo analiza la actuación del director "en foco" sin que este pueda intervenir, aunque sí puede escuchar cada comentario. Solo una vez llevado a cabo el análisis y tras oír su reacción al feedback recibido, el grupo procede a sugerir consejos u otros cursos posibles de acción. La sesión concluye con una síntesis del proceso seguido que inicia el coordinador. Como puede verse, es el grupo quien ofrece feedback contextualizado al participante «en foco». En todo momento el coordinador orienta y regula ese feedback para garantizar dos objetivos: ampliar el horizonte de posibilidades de los participantes sobre 
su práctica directiva (Aas \& Vavik, 2015) y reflexionar sobre el ajuste entre las competencias de liderazgo que los participantes poseen y las competencias que deberían poseer dadas las características de los centros escolares a su cargo (Passmore \& Law, 2009).

La sesión 7 se celebra pasadas unas semanas desde la sesión 6 y constituye la última parte del programa.
Se trata de una sesión de seguimiento de las mejoras introducidas en el ejercicio de su liderazgo a partir de la discusión grupal durante el programa. De nuevo, la relación entre el perfil de liderazgo de los participantes y las características organizativas de sus escuelas constituye el foco principal de las mejoras que deben planificar.

\section{Figura 1}

La estructura del programa de formación
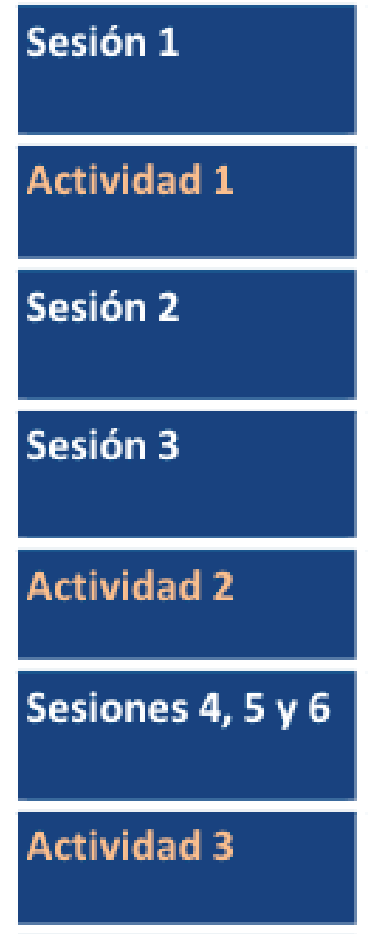

Sesión 7
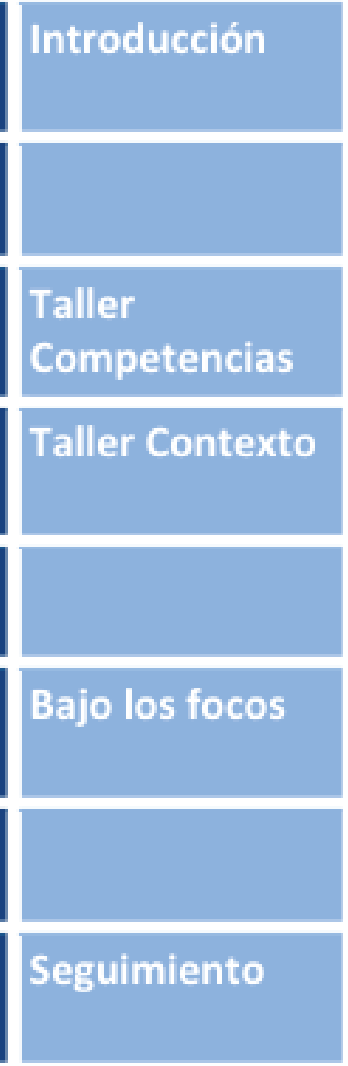

\section{- Introducción al programa formativo \\ - Constituir el grupo}

- Completar el Perfil de Competencias para la Dirección Escolar

- Reflexión individual sobre los resultados y sus consecuencias

\section{- Analizar los perfiles de los participantes \\ - Fortalezas y desventajas en el contexto de cada participante}

\section{- Información sobre el centro escolar de los participantes}

- Adecuación entre el perfil y el contexto organizativo

- Elaborar un caso: narrar una situación problemática experimentada en el ejercicio de la dirección escolar

\section{- Análisis grupal de los casos \\ - Cada participante "bajo los focos" \\ - Elaboración de un plan de mejora \\ - Puesta en práctica y diario reflexivo}

\section{- Análisis de la puesta en práctica de los planes de mejora \\ - Conclusiones y síntesis de lo aprendido}

\section{Instrumentos de obtención de información}

La recogida de información se llevó a cabo mediante metodología mixta de investigación. Se administraron cuestionarios en diferentes momentos del desarrollo del programa para evaluar su eficacia, concretamente: (a) percepciones sobre la experiencia «bajo los focos» al final de las sesiones 3 a 5; (b) expectativas y motivación al inicio del programa; y (c) «Cuestionario Final de Satisfacción FORDICO» al terminar la sesión 7.
Este último es una escala tipo Likert de 5 valores y 48 ítems estructurada en 3 partes: (1) desarrollo del programa, (2) impacto del programa y (3) valoración general del programa. El instrumento fue diseñado siguiendo el modelo de cuestionarios validados sobre satisfacción de participantes en otros procesos de formación (Aas \& Flückiger, 2016; De-Miguel et al., 2011; Flückiger et al., 2016). Antes de su distribución fue sometido a validación de contenido mediante juicio de expertos. Una vez recogida la información, 
se determinó su fiabilidad mediante el estadístico Alpha de Cronbach y su validez mediante la prueba $\mathrm{h}$ de Kruskal-Wallis para contraste no paramétrico de dos muestras independientes medidas en una escala ordinal. Este último denota, con más del $99 \%$ de confiabilidad, que no existen diferencias estadísticamente significativas entre las opiniones de los partici-

\section{Tabla 1}

Resultados del "Cuestionario de satisfacción FORDICO" pantes de diferentes grupos respecto a las tres escalas ( $p>0.05$ en cada escala). Los resultados de ambas pruebas se sintetizan en la Tabla 1. Finalmente, se realizó un análisis descriptivo de cada una de las escalas del cuestionario que podrá verse en la sección de resultados.

\begin{tabular}{|c|c|c|c|c|c|c|}
\hline \multirow{2}{*}{ Escalas } & \multirow{2}{*}{ Fiabilidad } & \multirow{2}{*}{$\mathbf{X}$} & \multirow{2}{*}{$\sigma$} & \multicolumn{3}{|c|}{ H índice de Kruskal-Wallis } \\
\hline & & & & Chi cuadrado & fd & $\begin{array}{c}\text { sig } \\
\text { asintótico }\end{array}$ \\
\hline $\begin{array}{l}\text { Desarrollo del programa } \\
\text { (15 ítems) }\end{array}$ & .892 & 4.8 & .39 & 14.442 & 12 & .273 \\
\hline Impacto (25 ítems) & .936 & 4.6 & .51 & 16.247 & 12 & .180 \\
\hline Evaluación general (8 ítems) & .858 & 4.57 & .65 & 15.085 & 12 & .237 \\
\hline
\end{tabular}

En cuanto a los métodos cualitativos, las siete sesiones de formación desarrolladas por cada uno de los grupos de directores/as fueron grabadas en video -290 horas de grabación- y sometidas a análisis de contenido con ayuda del programa de análisis de datos cualitativos MAXQDA12.

El análisis utilizó diferentes sistemas de análisis en función de los distintos propósitos del estudio. En primer lugar, se diseñó un sistema de categorías agrupadas en cuatro dimensiones: actuaciones del coach, actuaciones de los participantes, desarrollo de las sesiones y conocimientos tácitos y explícitos del participante. Estas dimensiones y categorías perseguían: (a) analizar la dinámica grupal en profundidad; (b) realizar una evaluación criterial de la interacción en los grupos en función del grado de cumplimiento de los obje- tivos y la estructura del programa de formación; y (c) analizar el conocimiento práctico construido por cada grupo durante su proceso formativo. La Figura 2 representa el sistema completo de categorías de análisis y puede ser consultada en el enlace https://figshare. com/s/e03e1d9583e211e6edb1.

En segundo lugar, se llevó a cabo un análisis de los sesgos y las dificultades que afrontaron los grupos en el curso de su interacción, mediante un instrumento adaptado por el equipo de investigación a partir de otro de Schippers et al. (2014). En concreto, el instrumento (Figura 3), que puede verse en el enlace https:// figshare.com/s/a3cb996bd2397e9e1a13, nos permitió analizar: las condiciones estructurales relativas a la composición y dinámica de los grupos; las dificultades para reunir información relevante; los sesgos en 
el procesamiento de la información; y los sesgos que impidieron lograr una reflexión profunda. También se analizaron los recursos y las estrategias que algunos grupos utilizaron para remediar esas dificultades.

En tercer y último lugar, para profundizar en la construcción del conocimiento práctico generado por los directores participantes en el programa de formación, sometimos el contenido grabado de las sesiones del programa a un doble análisis mediante sendos marcos conceptuales. Para el análisis primario, se volvió a analizar la información que habíamos codificado bajo la dimensión "Conocimientos tácitos y explícitos del participante (CON)" del sistema de análisis de la Figura 2. Las categorías contempladas inicialmente -Metáforas, Creencias, Emociones, Dificultades, Conocimientos explícitos, Prácticas consolidadas y Biografía- fueron enriquecidas con las del sistema planteado por Poggi (2001). El sistema resultante incluyó: (a) dificultades habituales o propias de la práctica profesional; (b) 'representaciones imaginarias', incluidas metáforas, emociones asociadas a situaciones experimentadas, y aspectos relevantes de su biografía profesional; y (c) reglas y principios de la práctica como creencias, conocimientos y prácticas consolidadas. Sin embargo, los ejes temáticos o las áreas de la práctica a los que se refería el conocimiento práctico producido/sintetizado por los grupos en el curso de su interacción quedaban fuera de este análisis. Para relevarlo llevamos a cabo un análisis secundario de los datos mediante una adaptación (Figura 4) del marco conceptual de Lazaridou (2008). Esta parte del análisis se presenta de modo más extenso en Sánchez-Moreno et al. (2019).

\section{Figura 4}

Marco conceptual del análisis secundario de los datos

1.1. Limitaciones externas e internas

1.2. Complejidad del trabajo

1. Conocimiento acerca de la organización
1.3. Conocimiento de la administración: Leyes, normativas y recursos de que se dispone

1.4. Administración del tiempo

2.1. Los intereses en juego y los conflictos que provocan los intereses contrapuestos

2. Conocimiento acerca de las 2.2. Conocimiento de sí mismo y reflexiones sobre el proceso de construcción de su identidad como directores.

2.3. Fortalezas y debilidades de los miembros de la organización 2.4. Conocimientos y habilidades para entablar relaciones productivas

\section{Conocimiento de las tareas 3.1. Funciones y responsabilidades y perfil de liderazgo requerido}
4. Emociones
4.1. Emociones

Nota: Adaptado de Lazaridou (2008, pp. 6-9). 


\section{Resultados}

\section{Grado de satisfacción general con el programa}

Las respuestas al "Cuestionario de Satisfacción FORDICO” hablan de un alto grado de satisfacción entre los participantes. Se obtuvieron puntuaciones entre 4,57 y 4,8 (sobre 5) en las tres partes del cuestionario: desarrollo (15 ítems), impacto (25 ítems) y evaluación general del programa (8 ítems). En todos ellos, la desviación típica $(\sigma)$ fue menor a 1 (sobre un máximo de 2), lo que evidencia una alta concentración de las respuestas de los participantes. En otro artículo publicado sobre esta investigación se puede apreciar un análisis más extenso de la información cuantitativa recogida (Oliva-Rodríguez \& López Yáñez, 2019).

\section{Dinámica de los grupos}

El aspecto que más influyó en el funcionamiento y la dinámica de los grupos de formación fue su grado de cumplimiento de la secuencia de aprendizaje promovida por el programa, algo sobre lo que abundaremos en la siguiente sección. Esto no es sorprendente teniendo en cuenta que esa secuencia fue diseñada con el fin de promover eficazmente la reflexión, una de las principales condiciones para mejorar la práctica del liderazgo. Más concretamente, se observó que la profundidad y riqueza de las reflexiones de los participantes sobre su práctica y la de los demás, durante las sesiones 4 a 6 ('en foco'), dependía en gran medida de que hubiera una progresión adecuada del grupo a lo largo de los diferentes tipos de preguntas previstas en la estructura del programa. Al principio, las preguntas "puntuales" permitían trazar una imagen general de la situación y relacionarla con las características y circunstancias del contexto. Posteriormente, las preguntas "horizontales" tendían a orientar la reflexión hacia la forma en que cada líder ejercía su rol. A modo de ejemplo: “... ¿cómo distribuyes las competencias entre los miembros de la escuela [... confías en cada persona o estás controlando todo?”(15.2). Finalmente, las preguntas "verticales" ayudaban a los participantes a tomar conciencia de su comportamiento y de los pensamientos y sentimientos que los empujaron a adoptar tal comportamiento. Ocasionalmente, también permitieron pensar en acciones que se podrían haber ejecutado alternativamente.

La cantidad y variedad de preguntas fueron cruciales para lograr una información rica y densa sobre la cual construir el análisis. En varios grupos, la falta de cumplimiento de la secuencia de preguntas bloqueó la reflexión sobre las circunstancias del caso y también obstaculizó la búsqueda de conductas alternativas. Los participantes 'en foco' generalmente se sentían decepcionados con las evaluaciones prematuras de su comportamiento, especialmente si tales evaluaciones no se basaban en una recopilación previa de información suficiente y relevante. Un efecto similar tuvieron las digresiones respecto de la situación analizada, ya que frecuentemente distraían al grupo del objeto de análisis, que no era otro que la práctica de liderazgo del participante en foco. Este tipo de comentarios derivaba fácilmente hacia aspectos generales de la vida escolar en los que todo líder escolar, incluso todo maestro, puede reconocerse fácilmente. Sin embargo, esos comentarios, generalmente acompañados de conclusiones y consejos que supuestamente deberían funcionar en cualquier situación, distraían al grupo del análisis de las particularidades del caso bajo análisis y lo mantenían en una zona de consenso básico, alejándolo de la tarea, más arriesgada socialmente, de proponer y recibir preguntas y críticas.

Algo similar ocurría cuando el análisis del caso se centraba en la actuación de otros agentes involucrados, en lugar de hacerlo sobre al líder 'en foco'. A diferencia de esto, los grupos productivos claramente centraron el análisis en el participante 'en foco' y evitaron digresiones sobre el comportamiento de los otros actores que podrían haber ocultado los errores potenciales del líder e impedido la búsqueda de alternativas.

Cada sesión finalizaba con una síntesis entre todos los participantes, tanto de las lecciones aprendidas en términos de fortalezas y debilidades de su comportamiento, como de las posibles mejoras, especialmente aquellas que fueron el centro de atención. Pues bien, se 
registraron niveles más altos de reflexión entre los integrantes de aquellos grupos donde se logró completar la secuencia de aprendizaje. Por otro lado, casi tan importante como el cumplimiento preciso de la secuencia de aprendizaje resultaron las intervenciones que relacionaban el comportamiento de los directivos bajo el foco, su perfil como líderes y las características organizativas de sus escuelas (Neufeld \& Roper, 2003).

Por último, el establecimiento de un clima de confianza en el grupo resultó otro aspecto clave para el logro de una dinámica de aprendizaje exitosa. Tal clima de confianza facilitó a los participantes tanto recibir como dar críticas e incluso opiniones negativas entre sí, sin provocar molestias o malestar. Esta actitud de crítica constructiva resultó decisiva para que el grupo profundizara en el análisis de los casos. Cuando solo había valoraciones positivas, la reflexión sobre la propia práctica fue mucho menor y la dinámica del grupo adoptaba la pauta de una terapia informal.

\section{Ajuste de los grupos a la estructura del programa}

Durante el análisis, clasificamos a los grupos según la fidelidad con la que se ajustaron a la estructura establecida por programa de formación. Establecimos para esto tres tipos que denominamos 'ajuste máximo', 'ajuste mínimo' y 'ajuste parcial'. En la Tabla 2 -que puede consultarse en el enlace https://figshare. com/s/4154a560c77edf8962ff- se muestran los criterios utilizados, así como el ajuste, desajuste o ajuste parcial de 6 grupos seleccionados como los más representativos de esos tres tipos.

El análisis del proceso de coaching grupal de los 6 grupos clasificados bajo la tipología de la Tabla 2 evidenció diferencias relevantes en sus procesos de aprendizaje. Los grupos 05 y 07 fueron los que experimentaron un proceso de reflexión más profundo, lo cual pudo ser asociado a su mayor ajuste a la estructura y a los objetivos marcados por el programa. Igualmente determinantes fueron las actuaciones del coordinador y la predisposición de los participantes a reflexionar sobre sus actuaciones. El resultado fue que los participantes reconocieron haber logrado aprendizajes y admitieron haber experimentado cambios en el desempeño del cargo en mayor medida que los del resto de grupos.

La observación en profundidad del funcionamiento de estos dos grupos demostró lo determinante que resulta la actitud del coach en el funcionamiento de los grupos de formación. Los de estos grupos desplegaron la variedad de roles que sugiere la literatura especializada (Reischmann, 2017; Youde, 2018): guía a través de la estructura del programa, facilitador y animador de la comunicación, moderador de las discusiones, al tiempo que ofrecían consejos, formulaban preguntas o establecían conclusiones como los demás participantes. Resultaron determinantes sus esfuerzos para mantener la interacción grupal dentro de la secuencia y ajustada a los tiempos previstos por el programa, en particular cuando se producían digresiones o desvíos de la temática que se estaba tratando. También los esfuerzos para promover y mantener un clima de confianza que permitiera la comunicación franca y la crítica constructiva (Giannoukos et al., 2015; Peiser et al., 2018).

Por el contrario, los grupos 01 y 15 resultaron mucho menos productivos que los anteriores, lo cual se asoció a su menor seguimiento de la estructura del programa. En estos casos los coordinadores tuvieron un papel mucho menos activo. En el caso de los grupos 02 y 16 tampoco encontramos un esfuerzo destacable por parte de los coordinadores por seguir la estructura del programa y perseguir sus objetivos. Sin embargo, esa estructura se mantuvo y los objetivos se lograron moderadamente gracias al compromiso que adoptó uno de los participantes de cada uno de esos grupos, convirtiéndose en el verdadero líder en la sombra. Un desarrollo ampliado de estos resultados puede verse en Sánchez-Moreno et al. (2019).

\section{Sesgos y dificultades de dos grupos durante el proceso formativo}

A continuación, se realizó un análisis secundario de los datos mediante el instrumento -elaborado inicialmente por Schippers et al. (2014) y reelaborado por nosotros-, a fin de detectar los sesgos y las dificultades que afectaban a: las condiciones estructurales relativas 
a la composición y dinámica de los grupos; las dificultades para reunir información relevante; los sesgos en el procesamiento de la información; y los sesgos que impiden lograr una reflexión profunda. También se analizaron los recursos y las estrategias que los grupos utilizaron para remediar esas dificultades. Para eso se tomaron dos grupos representativos, el número 15 clasificado en la categoría de 'ajuste mínimo’ y el 05, clasificado en la de 'ajuste máximo' a la estructura y a los objetivos del programa de formación.

El grupo 15 reflejó la mayoría de los sesgos y las dificultades previstos en el sistema de análisis, concretamente: superficialidad; dispersión; elaboración insuficiente; encuadre inadecuado o parcial; falta de profundización o de pensamiento estratégico; ilusiones o distorsiones de la realidad; y sesgo confirmatorio. Así mismo, en cuanto a los aspectos estructurales, mostró falta de claridad en los objetivos y un rol del coach como mero administrador del tiempo disponible. A cambio, el grupo -y en particular el coach-consiguió crear un clima de respeto y seguridad psicológica que facilitó la reflexión.

Sin embargo, el grupo 05, si bien también mostró algunos de los sesgos y las dificultades antes mencionados, puso en marcha un buen número de las estrategias para remediar las dificultades previstas en el sistema de análisis. Algunas de estas estrategias consiguieron que la información recopilada fuera relevante y útil: se evitaron juicios a priori; se delimitó adecuadamente el problema; y se solicitó información más detallada mediante una variedad de tipos de pregunta. Otras garantizaron un procesamiento adecuado de la información: se puso en relación la información disponible, se sintetizó y se formularon conclusiones relevantes; y se establecieron implicaciones para la práctica, incluyendo consejos o recomendaciones. Finalmente, otras estrategias proyectaron al grupo hacia un pensamiento estratégico, aunque fueron las menos usadas: en alguna ocasión, el coach planteó al grupo un encuadre alternativo al implícito inicialmente en la descripción de los hechos y en otras intentó -con desigual éxito-que el grupo adoptara un pensamiento sistémico, mirando más allá de las intenciones o predisposiciones psicológicas de los actores.
Sin embargo, solo en ocasiones aisladas consiguió el grupo 15 poner en marcha alguna de las estrategias mencionadas en el párrafo anterior. Esto explica la diferencia en los logros de ambos grupos en cuanto a la profundidad de su reflexión y sus conclusiones. Además, proporciona evidencia empírica acerca de los aspectos clave que impulsan o bien dificultan la reflexividad en los grupos en formación, en concreto aquellos que implican a líderes en formación en el análisis de sus prácticas y sus perfiles directivos.

Por otro lado, el grupo 05, el más exitoso de los dos, fue capaz de reintroducir la información procedente de los instrumentos previstos en el programa de formación en su proceso reflexivo, algo que diversos autores han destacado como clave del éxito en programas de formación similares (Aas \& Vavik, 2015; Lochmiller, 2014; Passmore \& Law, 2009). Así mismo, el coach se reveló como la pieza fundamental que permitió al grupo evitar las dificultades y los sesgos comunes que amenazan el proceso reflexivo y adoptar estrategias adecuadas para remediarlos, como otros autores han puesto también de manifiesto (Britton, 2015; Dyke, 2014; Thornton, 2010).

La construcción de conocimiento sobre la dirección escolar en los grupos

Como ya se ha mencionado, a lo largo de las siete sesiones del programa, los participantes analizaron su conducta y su perfil de competencias como líderes escolares. Reflexionaron sobre su propia práctica y la de sus compañeros mientras discutían posibles alternativas a las decisiones que habían adoptado como líderes escolares. Nuestra misión era capturar el conocimiento implícito sobre su práctica profesional que emergía de esa discusión.

Los directores escolares participantes en el programa formativo identificaron a la administración -en concreto la inspección escolar- como la principal fuente de limitaciones de su acción directiva. Bajo la metáfora 'con la Iglesia hemos topado', un participante aludió a la autoridad jerárquica, que algunos percibieron a menudo arbitraria: "dicen una cosa hoy y otra mańana, o se contradicen los unos a los otros: 
son reinos de taifas" (03.6); "esta misma administración que nos abre la puerta nos va poniendo picas en el camino..." (03.2).

Algunas metáforas bastante expresivas condensaron la complejidad de su práctica profesional, así como las perturbaciones a las que se ve sometida. En ellas, por ejemplo, el director aparece como un capitán y el centro escolar como un barco $(05.2,05.4)$, donde todos deben remar acompasados y manejar bien la ola (03.3). El rol directivo está sometido a sobrecarga de tareas y necesita actuar con tacto y parsimonia. La gestión burocrática fue señalada como otra carga limitante de las posibilidades de una acción más autónoma, mostrándose divididos entre la aceptación y la resistencia a que este tipo de tareas se conviertan en predominantes.

El conocimiento acerca de las personas y las relaciones resultó el principal foco de interés de la reflexión dentro de los grupos. Algunas de las metáforas recogidas señalaron este aspecto como sujeto a frecuente confrontación: "Es muy difícil conseguir en nuestro trabajo (...) un nido de paz de tres cuartos de hora"(05.4), y al despliegue de estrategias micropolíticas por parte de los diferentes actores. Otras metáforas señalaron la necesidad de conocer bien a la comunidad educativa, en especial a las familias de los estudiantes, para conseguir de ellos una participación más profunda.

En definitiva, de manera generalizada la construcción de relaciones sociales satisfactorias y productivas en su escuela resultó la principal preocupación de los directivos en formación. Nuestros registros contienen conocimiento relevante acerca de cómo fomentar un clima social satisfactorio -y sobre las dificultades que deben ser afrontadas-, incluyendo el fomento del trabajo en equipo, la construcción de un sentimiento de comunidad o la gestión de los significados culturales. También capturamos la preocupación por organizar modalidades de trabajo productivas y eficaces, que movilizó el conocimiento de los participantes acerca de la delegación de responsabilidades y la participación en las decisiones.

Otro aspecto importante de su conocimiento profesional se refería al conocimiento acerca de ellos mismos y de los cambios operados en el ejercicio de su liderazgo, algo que frecuentemente aparecía asociado a la reflexión sobre sus trayectorias biográficas. Este tipo de conocimiento incluía en algunos casos una percepción más rica y autocrítica sobre las emociones que desplegaron. En concreto, muchos de los participantes declararon haber aprendido a cuidarse emocionalmente, reconociendo que ahora experimentan satisfacción ante situaciones difíciles que antes les producían una gran inquietud (09.5). Se ha registrado que los directivos manifiestan haber tenido miedo al principio de su ejercicio como director/a; pero también fueron registradas expresiones de alegría asociadas al sentimiento de logro tras llevar a cabo procesos de cambio efectivos o bien mejorar las relaciones entre diferentes colectivos (03.2, 03.3, 03.4, 03.5). Los procesos ante los que con más frecuencia manifestaron tristeza estaban asociados en nuestros registros a la impotencia por lograr una colaboración docente más estrecha (06.7) o a la falta de autonomía para tomar decisiones que consideraban relevantes.

En cuanto al conocimiento de las tareas, aquí prevaleció la sensación de sobrecarga, lo que sugería para algunos la necesidad de priorizar y de adaptar su propio perfil de liderazgo a las condiciones reales de trabajo. La planificación y la innovación aparecieron como tareas cruciales de la acción directiva.

\section{Conclusiones}

El desarrollo profesional de los/as directores/ as escolares necesita estrategias bien fundamentadas sobre los principios teóricos de la andragogía, que a la vez exploten y desarrollen el conocimiento práctico adquirido durante su desempeño. Esta investigación diseñó, puso a prueba e implementó, durante un periodo de cinco años, un programa de coaching grupal que tuvo como ejes la retroalimentación y el análisis de la práctica. El resultado es un programa de formación de líderes escolares altamente estructurado que ha probado su eficacia y que se encuentra listo para ser usado por cualquier administración educativa, con las adaptaciones pertinentes. 
Por otro lado, la investigación llevada a cabo en paralelo al desarrollo del programa formativo ha permitido sistematizar $-y$ codificar en un manual de uso, también disponible- información altamente relevante sobre los requisitos, los criterios y las prácticas que garantizarán el éxito del programa en futuras ediciones. En concreto: (a) los sesgos y las dificultades que algunos grupos afrontan en su proceso comunicativo, la manera en que los sesgos pueden ser corregidos y las dificultades superadas; (b) la relevancia que tiene seguir la secuencia de aprendizaje prevista en la estructura del programa, así como el papel que desempeñan todos sus elementos: los instrumentos para proveer retroalimentación a los usuarios sobre su perfil de liderazgo y sobre las características de sus escuelas, los casos sobre situaciones problemáticas, las preguntas en sus diferentes modalidades, y los planes de mejora; y (c) el papel decisivo que tiene el coordinador del grupo de formación y, por tanto, la importancia de garantizar que los coaches reciban una formación específica que les habilite para conducir con éxito al grupo durante todas las fases del programa.

Sobre la formación para los coordinadores, esta debería abarcar tanto el conocimiento del programa y de sus fundamentos teóricos como las estrategias apropiadas para incentivar la reflexión y la construcción de conocimiento en los participantes. Los coordinadores deben contar con competencias que les permitan establecer relaciones entre el conocimiento práctico de estos y el conocimiento teórico disponible acerca del liderazgo educativo (Heslin \& Keating, 2017); pero también deben disponer de habilidades sociales para mantener la cohesión del grupo y su disposición para la reflexión abierta y en profundidad; habilidades como la escucha activa, la empatía o la asertividad (O’Mahony \& Barnett, 2008). Un desarrollo ampliado del papel de coach en este programa de formación puede verse en Altopiedi \& Burgos (2020).

Otra aportación relevante del estudio ha sido los instrumentos de análisis de la interacción grupal utilizados en la investigación, los cuales han resultado eficaces en sus diferentes propósitos y, por tanto, podrían utilizarse en futuros estudios. En particular, el adaptado a partir de Schippers et al. (2014), podría utilizarse además para orientar el diseño de versiones futuras del programa de formación, con el objeto de garantizar las condiciones estructurales que se han mostrado necesarias para facilitar la reflexividad grupal. Aún más, también podría ser útil para orientar la formación del coordinador de programas como este, para hacerlos más hábiles a la hora de identificar las dificultades comunicativas, así como para poner en marcha estrategias eficaces de superación de estas dificultades.

Finalmente, el repertorio de conocimientos prácticos que se han recogido puede servir de base, a este o a futuros programas de formación continua de directivos escolares, para favorecer la reflexividad de los participantes. Los formadores de estos programas podrían utilizar las dificultades identificadas por los participantes en este estudio, sus representaciones imaginarias y sus reglas y principios de la práctica para provocar ciclos de reflexión en los directivos en formación y, a la vez, para sacar a la luz el conocimiento práctico que ellos mismos poseen.

Como limitaciones del estudio, se señalan principalmente dos. En primer lugar, la eficacia del programa se determinó en base a la satisfacción de los participantes. Hubiera sido útil -y se recomienda para futuros estudios- una evaluación del impacto real del programa sobre la práctica de los participantes como líderes escolares, mediante metodologías como la observación de la práctica o las entrevistas en profundidad meses después de la finalización del programa, incluyendo entrevistas a otros miembros del equipo directivo y docente. En segundo lugar, el análisis general del proceso de coaching grupal se centró en seis de los 14 grupos participantes, mientras que el de los sesgos y las dificultades ocupó a dos de esos grupos que, no obstante, eran representativos de los tres tipos que se establecieron según su ajuste a la estructura del programa. El análisis del material grabado en las 7 sesiones de los 14 grupos probablemente habría aportado un detalle mayor en los resultados y las conclusiones. 


\section{Notas}

1) "Professional Learning through Reflection promoted by Feedback and Coaching", Comenius Lifelong Learning Multilateral Projects. Coordinador: Prof. Dr. Stephan Gerhard Huber. Referencia: 528356-LLP-1-2012-1-CH-COMENIUS-CMP.

2) Sánchez-Moreno, M. \& López-Yáñez, J. (Dirs.). (2015-2017) Formación de directores de instituciones de formación primaria, secundaria y superior basada en el análisis de la práctica y el coaching. Ministerio de Economía y Competitividad. Convocatoria de proyectos de $\mathrm{I}+\mathrm{D}+\mathrm{i}$. Referencia EDU2014-53175-P.

\section{Referencias}

Aas, M., \& Flückiger, B. (2016). The role of a group coach in the professional learning of school leaders. Coaching: An International Journal of Theory, Research and Practice, 9(1), 38-52. https:// doi.org/10.1080/17521882.2016.1143022

Aas, M., \& Vavik, M. (2015). Group coaching: A new way of constructing leadership identity?, School Leadership \& Management, 35(3), 251-265. https://doi.org/10.1080/13632434. 2014.962497

Altopiedi, M. \& Burgos, A. (2020). El papel del coach en la formación de directivos: análisis de un programa específico. Educar, 56(1), 183-199. https://educar.uab.cat/article/view/v56-n1-altopiedi-burgos

Bagnall, R. (2017) A critique of Peter Jarvis's conceptualisation of the lifelong learner in the contemporary cultural context. International Journal of Lifelong Education, 36, 1-2.

Begley, P.T. (2006). Self-knowledge, capacity, and sensitivity: Prerequisites to authentic leadership by school principals. Journal of Educational Administration, 44(6), 570-589. https://doi. org/10.30827/profesorado.v23i2.9390

Bloom, G., Castagna, C., Moir, E., \& Warren, B. (2005). Blended Coaching: Skills and strategies to support principal development. Corwin Press.
Botha, R.J.N. (2014). Knowledge Beliefs and Problem-solving Capabilities among South African School Principals. Journal of Social Sciences, 38(3), 247-253. https://doi.org/10. 30827/profesorado.v23i2.9390

Britton, J.J. (2015). Expanding the coaching conversation: Group and team coaching. Industrial and Commercial Training, 47(3), 116-120. https:// doi.org/10.1108/ICT-10-2014-0070

Camburn, E.M. \& Han, S.W. (2017). Teachers' professional learning experiences and their engagement in reflective practice: a replication study. School Effectiveness and School Improvement, 28(4), 527-554.

Cosner, S., Walker, L., Swanson, J., Hebert, M., \& Whalen, S.P. (2018). Examining the architecture of leadership coaching: Considering developmental affordances from multifarious structuring. Journal of Educational Administration, 56(3), 364-380.

Creasy, J., \& Paterson, F. (2015). Leading coaching in schools. National College for School Leadership. Cullen-Lester, K., Maupin, C., \& Carter, D. (2017). Incorporating social networks into leadership development: A conceptual model and evaluation of research and practice. The Leadership Quarterly, 28, 130- 152.

Daly, A.J., Moolenaar, N.M., Bolivar, J.M., \& Burke, P. (2010). Relationships in reform: The role of teachers' social networks. Journal of Educational Administration, 48(3) 359-391.

Day, C., \& Sammons, P. (2013). Successful leadership: $A$ review of the international literature. CfBT Education Trust.

Day, C., Leithwood, K., \& Sammons, P. (2008). What we have learned, what we need to know more about. School Leadership \& Management, 28(1), 83-96.

De-Miguel, M., San-Fabián, J.L., Belver, J.L. \& Argüelles, M.C. (2011). Evaluación de la satisfacción de los participantes en formación profesional para el empleo. RELIEVE, 13(1), 83-103. https://doi.org/10.7203/relieve.13.1.4213 
Devos, G., \& Bouckenooghe, D. (2009). An Exploratory Study on Principals' Conceptions about Their Role as School Leaders. Leadership and Policy in Schools, 8(2), 173-196. https://doi. org/10.30827/profesorado.v23i2.9390

Dyke, P.R.V. (2014). Virtual group coaching: A curriculum for coaches and educators. Journal of Psychological Issues in Organizational Culture, 5(2), 72-86. https://doi.org/10.7203/relieve.13. 1.4213

Dyke, M. (2017) Paradoxes of a long life learning: An exploration of Peter Jarvis's contribution to experiential learning theory. International Journal of Lifelong Education, 36(1-2), 23-34.

Ellis, S., Carette, B., Anseel, F., \& Lievens, F. (2014). Systematic reflection: Implications for learning from successes and failures. Psychological Science, 23(1), 67-72.

Fernández-Sánchez, M.R. \& Valverde-Berrocoso, J. (2014). Comunidades de práctica: un modelo de intervención desde el aprendizaje colaborativo en entornos virtuales. Comunicar, 42, 97-105, http://dx.doi.org/10.3916/C42-2014-09.

Flückiger, B., Aas, M., Nicolaidou, M., Johnson, G., \& Lovett, S. (2016). The potential of group coaching for leadership learning. Professional Development in Education, 43(4), 612-629. https://doi.org/10 $.1080 / 19415257.2016 .1223736$

Forde, C., McMahon, M., \& Gronn, P. (2013). Designing individualised leadership development programmes. School Leadership \& Management, 33(5), 440-456. https://doi.org/10.1080/1363 2434.2013.813455.

Forde, C., McMahon, M., Gronn, P., \& Martin, M. (2013). Being a leadership development coach: A multi-faceted role. Educational Management Administration \& Leadership, 41(1), 105-119. https://doi.org/10.1177/1741143212462699

Giannoukos, G., Bessas, G., Galiropoulos, C., \& Hioctou, V. (2015) The andragogy, the social change and the transformative learning educational approaches in adult education. Journal of Education and Practice, 6(7), 131-136.
Goff, P., Guthrie, J.E., Goldring, E., \& Bickman, L. (2014). Changing principals' leadership through feedback and coaching. Journal of Educational Administration, 52(5), 682-704, https://doi. org/10.1108/JEA-10-2013-0113.

Grissom, J.A., Egalite, A.J., \& Lindsay, C.A. (2021) How Principals Affect Students and Schools. A Systematic Synthesis of Two Decades of Research. The Wallace Foundation. http://www.wallacefoundation.org/principalsynthesis.

Hallinger, P., \& Bridges, E. (2017). A Systematic Review of Research on the Use of Problem-Based Learning in the Preparation and Development of School Leaders. Educational Administration Quarterly, 53(2), 255-288.

Hallinger, P., \& Snidvongs, K. (2005). Adding value to school leadership and management. National College for School Leadership.

Hargrove, R. (2008). Masterful coaching. Jossey-Bass. Harris, A., Leithwood, K., Day, C., Sammons, P., \& Hopkins, D. (2007) Distributed leadership and organizational change: Reviewing the evidence. Journal of Educational Change, 8, 337-347.

Hernández-Sellés N., González-Sanmamed, M., \& Muñoz-Carril, P.C. (2014). La planificación del aprendizaje colaborativo en entornos virtuales. Comunicar, 42, 25-33, http://dx.doi. org/10.3916/C42-2014-02.

Heslin, P., \& Keating, L. (2017). In learning mode? The role of mindsets in derailing and enabling experiential leadership development. The Leadership Quarterly, 28, 367-384.

Huber, S.G. (2013). Multiple Learning Approaches in the Proffesional Development of School Leaders - Theoretical Perspectives and Empirical Findings on Self-assessment and Feedback. Educational Management Administration and Leadership, 41(4), 527-540. https://doi. org/10.30827/profesorado.v23i2.9390

Huber, S.G., \& Hiltmann, M. (2011). Competence Profile School Management (CPSM) - an Inventory for the Self-Assessment of School Leadership. Educational Assessment, Evaluation and Accountability, 23(1), 65-88. https://doi.org/10.30827/ profesorado.v23i2.9390 
Huff, J., Preston, C., \& Goldring, E. (2013). Implementation of a coaching program for school principals: Evaluating coaches' strategies and the results. Educational Management Administration \& Leadership, 41(4), 504-526. https:// doi.org/10.1177/1741143213485467

Illeris, K. (2017) Peter Jarvis and the understanding of adult learning, International Journal of Lifelong Education, 36(1-2) 35-44.

Iranzo-García, P., Camarero-Figuerola, M., BarriosArós, C., Tierno-García, J. \& Gilabert-Medina, S. (2018). ¿Qué opinan los maestros sobre las competencias de liderazgo escolar y sobre su formación inicial? REICE. Revista Iberoamericana sobre Calidad, Eficacia y Cambio en Educación, 16(3), 29-48.

James-Ward, C. (2011). The Development of an infrastructure for a model of coaching principals. The International Journal of Educational Leadership Preparation, 6(1), 1-12. https://bit.ly/2OiYoz1

Knowles, M.S. (1984). Andragogy in action: Applying modern principles of adult learning. Jossey-Bass.

Knowles, M.S., Holton, E.F., \& Swanson, R.A. (2015). The adult learner: The definitive classic in adult education and human resource development (8th ed.). Routledge.

Konradt, U., Otte, K., Schippers, M.C., \& Steenfatt, C. (2016). Reflexivity in Teams: A Review and New Perspectives. The Journal of Psychology, 150(2), 153-174. https://doi.org/10.1080/002 23980.2015 .1050977$.

Kramer, R.M. (2018). Ingroup-Outgroup Trust: Barriers, Benefits, and Bridges. En Eric M. Uslaner (Ed), The Oxford Handbook of Social and Political Trust (pp. 95-118). https:// www.oxfordhandbooks.com/view/10.1093/ oxfordhb/9780190274801.001.0001/oxford hb-9780190274801-e-37

Lave, J., \& Wenger, E. (1991). Situated learning: Legitimate peripheral participation. Cambridge University Press.

Lazaridou, A. (2007). Values in principals' thinking when solving problems. International Journal of
LeadershipinEducation, 10(4),339-356.https:// doi.org/10.1080/13603120701381758

Lazaridou, A. (2008). The Kinds of Knowledge Principals Use: Implications for Training. International Journal of Education Policy and Leadership, 4(10), 1-15. https://files.eric.ed.gov/fulltext/ EJ898891.pdf.

Lochmiller, C.R. (2014). Leadership Coaching in an Induction Program for Novice Principals: A 3-Year Study. Journal of Research on Leadership Education, 9(1), 59-84. https://doi.org/ $10.1177 / 1942775113502020$

Lofthouse, R. (2019). Coaching in education: A professional development process in formation. Professional Development in Education, 45(1), 33-45.

López-Yáñez, J., Sánchez-Moreno, M., Altopiedi, M. \& Oliva-Rodríguez, O. (2018) Formación de directivos escolares mediante un programa basado en el coaching grupal, la retroalimentación y el análisis de la práctica. Profesorado. Revista de Curriculum y Formación del Profesorado. 22(1), 481-500. https://doi.org/10.30827/ profesorado.v22i1.9938

Merriam, S.B., \& Bierema, L. (2014) Adult Learning. Linking theory and practice. Jossey-Bass.

Ming Yu, W. (2018). Critical incidents as a reflective tool for professional development: An experience with in-service teachers. Reflective Practice, 19(6), 763-776.

Nestor-Baker, N.S., \& Hoy, W.K. (2001). Tacit Knowledge of School Superintendents: Its Nature, Meaning, and Content. Educational Administration Quarterly, 37(1), 86-129. https:// doi.org/10.1177/00131610121969253

Neufeld, B., \& Roper, D. (2003). Year II of collaborative coaching and learning in the effective practice schools: Expanding the work. Education Matters, Inc.

O’Mahony, G., \& Barnett, B. (2008). Coaching relationships that influence how experienced principals think and act. Leading and Managing, 14(1), 16-37. 
Oliva-Rodríguez, N. \& López-Yáńez, J. (2019). Autoaprendizaje en Pequeńos Grupos para la Formación de Directores Escolares. Análisis de un Programa Piloto. REICE. Revista Iberoamericana sobre Calidad, Eficacia y Cambio en Educación, 17(1), 77-93. https://doi.org/10.15366/ reice2019.17.1.004

Orr, M. (2011). Pipeline to preparation to advancement: Graduates' experiences in, throughout, and beyond leadership preparation. Education Administration Quarterly, 47, 114-172. https://doi.org/10.1177/0011000010378612

Orr, M., \& Orphanos, S. (2011). How graduate-level preparation influences the effectiveness of school leaders: A comparison of the outcomes of exemplary and conventional leadership preparation programs for principals. Education Administration Quarterly, 47(1), 18-70. https://doi. org/10.1177/0011000010378610

Passmore, J., \& Law, H. (2009). Cross-culture and Diversity in Coaching. En J. Passmore (Ed.), Diversity in Coaching: Working with Gender, Culture, Race and Age (pp. 4-15). Kogan Page.

Peiser, G., Ambrose, J., Burke, B., \& Davenport, J. (2018). The role of the mentor in professional knowledge development across four professions. International Journal of Mentoring and Coaching in Education, 7(1), 2-18.

Petridou, A., Nicolaidou M., \& Karagiorgi, Y. (2017). Exploring the impact of professional development and professional practice on school leaders' self-efficacy: a quasi-experimental study. School Effectiveness and School Improvement, 28(1), 56-73. https://doi.org/10.1080/092434 53.2016 .1236734

Piggot-Irvine, E., \& Youngs, H. (2011). Aspiring principal development programme evaluation in New Zealand. Journal of Educational Administration, 49(5), 513-541. https://doi.org/ $10.1108 / 09578231111159520$

Poggi, M. (2001). La formación de directivos de instituciones educativas. Algunos aportes para el diseño de estrategias. IIPE-UNESCO Sede Regional Buenos Aires.

Pont, B., Nusche, D. \& Moorman, H. (2009). Mejorar el liderazgo escolar. Volumen 1: Politica y práctica. OECD.

Pozo, J.I., Martín, E., Pérez-Echeverría, M.P., Scheuer, N., Mateos, M. \& De-la-Cruz, M. (2010). Ni contigo ni sin ti... Las relaciones entre cognición y acción en la práctica educativa. Infancia y Aprendizaje, 33(2), 179-184. https://doi. org/10.1174/021037010791114580

Reichard, R., Walker, D.O., Puter, S., Middleton, E., \& Johnson, S. (2017). Believing is becoming: The role of leader developmental efficacy in leader self-development. Journal of Leadership \& Organizational Studies, 24(2), 137-156.

Reischmann, J. (2017). Lifewide learning - Challenges for Andragogy. Journal of Adult Learning, Knowledge and Innovation, 1(1), 43-50.

Retna K.S. (2015). Different approaches to the professional development of principals: a comparative study of New Zealand and Singapore. School Leadership y Management, 35(5), 524-543. https://doi.org/10.1080/13632434. 2015.1107038

Robertson, J. (2008). Coaching educational leadership: Building leadership capacity through partnership. Sage.

Robinson, V.M., Lloyd, C.A., \& Rowe, K.J. (2008). The impact of leadership on student outcomes: an analysis of the differential effects of leadership types. Educational Administration Quarterly, 44(5), 635-674. https://doi.org/10.1177/ $0013161 X 08321509$

Rothes, A., Lemos, M., \& Gonçalves, T. (2017) Motivational profiles of adult learners. Adult Education Quarterly, 67(1), 3-29.

Salazar, M., Pazey, B., \& Zembik, M. (2013). What We've Learned and How We've Used It: Learning Experiences From the Cohort of a High-Quality Principalship Program. Journal of Research on Leadership Education, 8(3), 304-329. https:// doi.org/10.1177/1942775113502021 
Sánchez-Moreno, M., López-Yañez, J. \& Toussaint-Banville, M. (2019). Conocimiento práctico de directores escolares participantes en un dispositivo de formación. Profesorado. Revista de Curriculum y Formación del Profesorado. 23(2), 107-130. https://doi.org/10.30827/profesorado. v23i2.9390

Sawyer, A.J., Tomlinson, S.R., \& Maple, A.J. (2000). Developing essential skills through case study scenarios. Journal of Accounting Education, 18, 257-282. https://bit.ly/2Oak9BS

Schippers, M., Edmonson, A., \& West, M. (2014). Team Reflexivity as an Antidote to Team Information-Processing Failures. Small Group Research, 45(6), 731-769. https://doi.org/10. 1177\%2F1046496414553473

Schleicher, A. (2012). Preparing Teachers and Developing School Leaders for the 21st Century: Lessons from around the World. OECD Publishing.

Schön, D.A. (1991). The reflective practitioner: How professionals think in action. Basic Books.

Serrat, P.M. \& Badía, M.C. (2015). Profesorado novel y feedback del tutor: un estudio de casos. Profesorado: Revista de Curriculum y Formación del Profesorado, 19(1), 405-421.

Silva, P., Del-Arco, I. \& Flores, O. (2017). La formación de directores escolares en Cataluña: Lecciones aprendidas a cinco ańos del Decreto de Dirección. Bordón: Revista de Pedagogía, 70(1).

Spillane, J.P., \& Healey, K. (2010) Conceptualizing School Leadership and Management From a Distributed Perspective: An Exploration of Some Study Operations and Measures. The Elementary School Journal, 111(2), 253-281. https:// doi.org/10.30827/profesorado.v23i2.9390

Stoll, L., \& Kools, M. (2017). The school as a learning organisation: A review revisiting and extending a timely concept. Journal of Professional Capital and Community, 2(1), 2-17. https://doi. org/10.30827/profesorado.v23i2.9390

Thornton, C. (2010). Group and team coaching: The essential guide. Routledge.

Van Knippenberg, D., \& Schippers, M. (2007). Work group diversity. Annual Review of Psychology,
58, 515-41. https://doi.org/10.1146/annurev. psych.58.110405.085546

Walker, A., Bryant D.O., \& Lee, M. (2013). International patterns in principal preparation: Commonalities and variations in pre-service programmes. Educational Management Administration and Leadership, 41(4), 405-434. https:// doi.org/10.1177/1741143213485466

Walker, A., \& Smither, J.W. (2006). A five-year study of upward feedback: what managers do with their results matters. Personnel Psychology, 52(2), 393-423. https://doi.org/10.1111/ j.1744-6570.1999.tb00166.x

Weinstein, J., Azar, A., \& Flessa, J. (2018). An ineffective preparation? The scarce effect in primary school principals' practices of school leadership preparation and training in seven countries in Latin America. Educational Management Administration \& Leadership, 46(2), 226-257.

Wenger, E. (1998). Communities of Practice: Learning, Meaning, and Identity, Learning in Doing Social, Cognitive and Computational Perspectives. Cambridge University Press.

Whitmore, J. (2004). Coaching for performance: Growing people, performance, and purpose. Nicholas Brealey Publishing.

Wright, N., \& Adam, A. (2015). The 'critical friend' role in fostering reflective practices and developing staff cohesion: A case study in a new secondary school, New Zealand. School Leadership \& Management, 35(4), 441-457. https://doi.org/ 10.1080/13632434.2015.1070821

Youde, A. (2018) Andragogy in blended learning contexts: effective tutoring of adult learners studying part-time, vocationally relevant degrees at a distance, International Journal of Lifelong Education, 37(2), 255-272.

Zhang, W., \& Brundrett, M. (2010). School leaders' perspectives on leadership learning: The case for informal and experiential learning. Management in Education, 24(4), 154-158. https://doi. org/10.30827/profesorado.v23i2.9390 